\title{
Neoadjuvant Four-Drug Combination Therapy for NSCLC With EGFR Mutation Avoiding Total Pneumonectomy
}

\author{
Jingpei $\mathrm{Li}^{1,2,3}, \mathrm{Ke} \mathrm{Xu}^{1,2,3}$, Weipeng Cai ${ }^{1,2,3}$, Yalei Zhang ${ }^{1,2,3}$, Xin Zeng ${ }^{4}$, Fei Cui ${ }^{1,2,3}$, \\ Zhexue Hao ${ }^{1,2,3}$, Jun $\mathrm{Liu}^{1,2,3 *}$ and Jianxing $\mathrm{He}^{1,2,3 *}$ \\ 'Department of Thoracic Surgery, The First Affiliated Hospital of Guangzhou Medical University, Guangzhou, China, \\ ${ }^{2}$ Guangzhou Institute of Respiratory Health (GIRH), Guangzhou, China, ${ }^{3}$ State Key Laboratory of Respiratory Disease, \\ National Clinical Research Center for Respiratory Diseases, Guangzhou, China, ${ }^{4}$ Department of Pathology, The First Affiliated \\ Hospital of Guangzhou Medical University, Guangzhou, China
}

OPEN ACCESS

Edited by:

Yaxiong Zhang,

Sun Yat-sen University Cancer Center

(SYSUCC), China

Reviewed by:

Xuan Wu,

Peking University Shenzhen

Hospital, China

Dwight Hall Owen,

The Ohio State University,

United States

${ }^{*}$ Correspondence:

Jun Liu

liujun9707@sina.com

Jianxing $\mathrm{He}$

drjianxing.he@gmail.com

Specialty section:

This article was submitted to

Thoracic Oncology,

a section of the journal

Frontiers in Oncology

Received: 28 March 2020

Accepted: 08 June 2020

Published: 16 July 2020

Citation:

Li J, Xu K, Cai W, Zhang Y, Zeng X,

Cui F, Hao Z, Liu J and He J (2020)

Neoadjuvant Four-Drug Combination

Therapy for NSCLC With EGFR

Mutation Avoiding Total

Pneumonectomy.

Front. Oncol. 10:1145.

doi: 10.3389/fonc.2020.01145
We report a case of successful neoadjuvant four-drug combination therapy to avoid total pneumonectomy. A 33-year-old male patient was diagnosed with locally advanced non-squamous NSCLC harboring EGFR mutation in the left lower lobe. The patient experienced significant clinical downstaging after two cycles of neoadjuvant therapy, including icotinib, carboplatin, pemetrexed, and bevacizumab. He underwent a successful lobectomy avoiding pneumonectomy. The patient showed no recurrence in the follow-up of a chest computed tomographic scan, which is 17 months after surgery. The promising results of this neoadjuvant combination therapy provided a novel therapeutic option for patients with locally advanced EGFR-mutated NSCLC facing total pneumonectomy.

Keywords: neoadjuvant, EGFR mutation, targeted (selective) treatment, chemothearpy, surgery

\section{INTRODUCTION}

Even with multidisciplinary treatment, the 5-year overall survival rates in stage III NSCLC patients are $\sim 25$ to $35 \%$ (1). Molecularly targeted agents have been shown to improve the overall survival for patients with NSCLC harboring epidermal growth factor receptor (EGFR) mutation in the setting of advanced disease. Researchers have begun to seek the possibility of applying a single agent of EGFR- tyrosine kinase inhibitor (TKI) from advanced to earlier stage (2). However, despite achieving significant downstaging of tumors, local or distant disease relapsed in less than 1 year. Various combination therapy achieved the promising results in the setting of advanced stage, including chemotherapy/EGFR-TKI $(3,4)$, chemotherapy/Bevacizumab (5), EGFR-TKI/Bevacizumab (6, 7), and eventually chemotherapy/EGFR-TKI/Bevacizumab (8). Currently, some prospective trials (9-11) had investigated the critical role of EGFR-TKI (gefitinib or erlotinib) in neoadjuvant therapy, the objective response rate (ORR) was ranged from 42.1 to $54.5 \%$, and the rate of major pathological regression (MPR) was from $9.7 \%$ up to $24.2 \%$. Considering the neoadjuvant EGFR-TKI combined with chemotherapy or anti-angiogenesis was tolerable, and could improve the radical resection rate, so we proposed a four-agent combination therapy for locally advanced non-squamous non-small-cell lung cancer harboring EGFR mutations. 


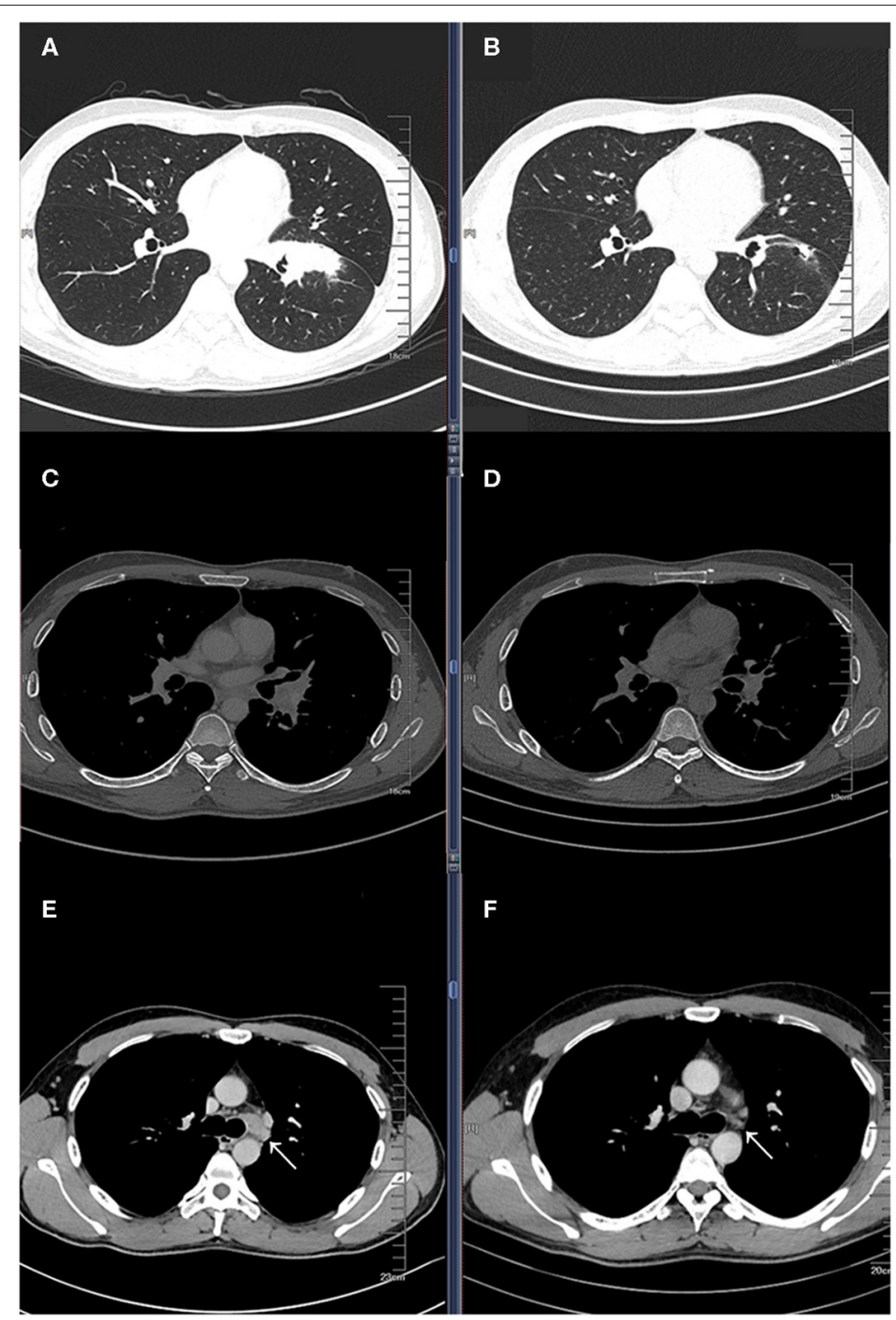

FIGURE 1 | Baseline computed tomography (CT) showing a lung mass in the left lower lobe pulmonary window (A) and mediastinal window (C), and an enlarged left lower paratracheal (4L) lymph node (E); CT images after two cycles of four drug combination therapy showing partial response in the pulmonary window (B) and mediastinal window (D), and a significantly reduction in the $4 \mathrm{~L}$ lymph node (F).

\section{CASE DESCRIPTION}

A 33-year-old male smoker presented with cough and sputum was admitted to the department of thoracic surgery. Computed tomography $(\mathrm{CT})$ revealed a lung mass in the left lower lobe (Figures 1A,C) and multiple hilar and mediastinal lymph nodes (Figure 1E). Cerebral magnetic resonance imaging and the bone scan did not show any lesion. The bronchoscopic biopsy confirmed the diagnosis of lung adenocarcinoma (cT2bN2M0, stage IIIA), and amplification refractory mutation system (ARMS)-polymerase chain reaction (PCR) showed an EGFR 19 exon deletion mutation. Multidisciplinary team discussion suggested neoadjuvant therapy over total pneumonectomy. Based on our previous experience of various combination therapies within our institute, four-drug combination therapy with fixed regimens was proposed, including icotinib $125 \mathrm{mg}$ po, tid, and intravenous carboplatin $150 \mathrm{mg}$ D1-D2, pemetrexed $800 \mathrm{mg} \mathrm{D} 1$, and bevacizumab $300 \mathrm{mg} \mathrm{D1}$, given a young age and good general status. After two cycles of neoadjuvant therapy, the Positron emission tomography/Computed tomography $(\mathrm{PET} / \mathrm{CT})$ revealed that the tumor was reduced from $4.6 \times$ $2.0 \mathrm{~cm}$ to $1.4 \times 1.1 \mathrm{~cm}$ (Figures 1B,D) with a normal standard uptake value (SUV), and there were no enlarged mediastinal or hilar lymph nodes (Figure 1F). No grade 3/4 adverse event (AE), including hemoptysis, was experienced,while rash and gastrointestinal symptoms were the most frequent AEs based on the patient's self-report.

The re-evaluation workup suggested a significant downstaging of the tumor (cT1bN0M0, stage IA2). Then 6 weeks after the last cycle of combination regimens were given, a left lower lobectomy was proposed. However, an occult parietal 


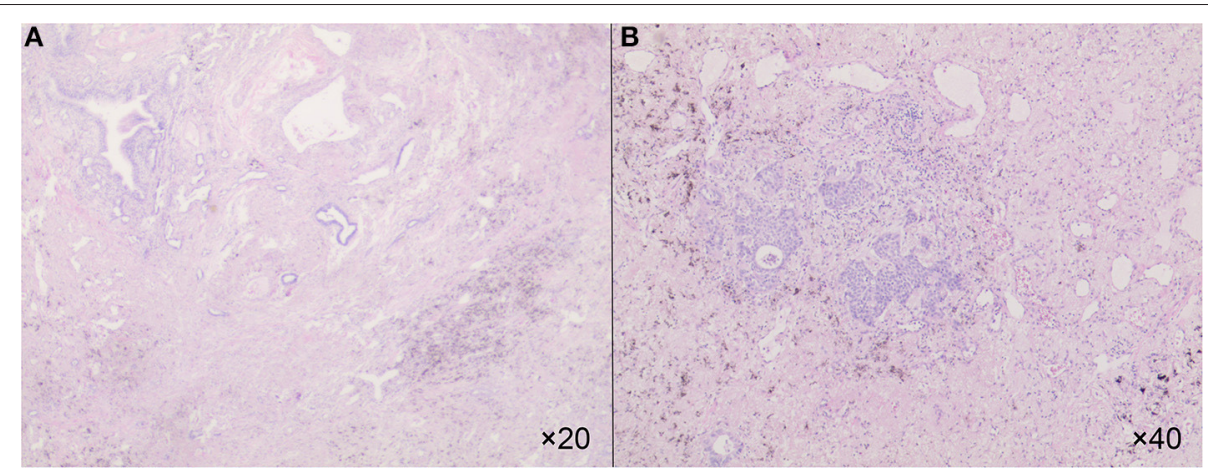

FIGURE 2 | The HE showed an increase in the number of residue cancer cells, but fibrosis still predominated in $20 \times(\mathbf{A})$ and in $40 \times(\mathbf{B})$.

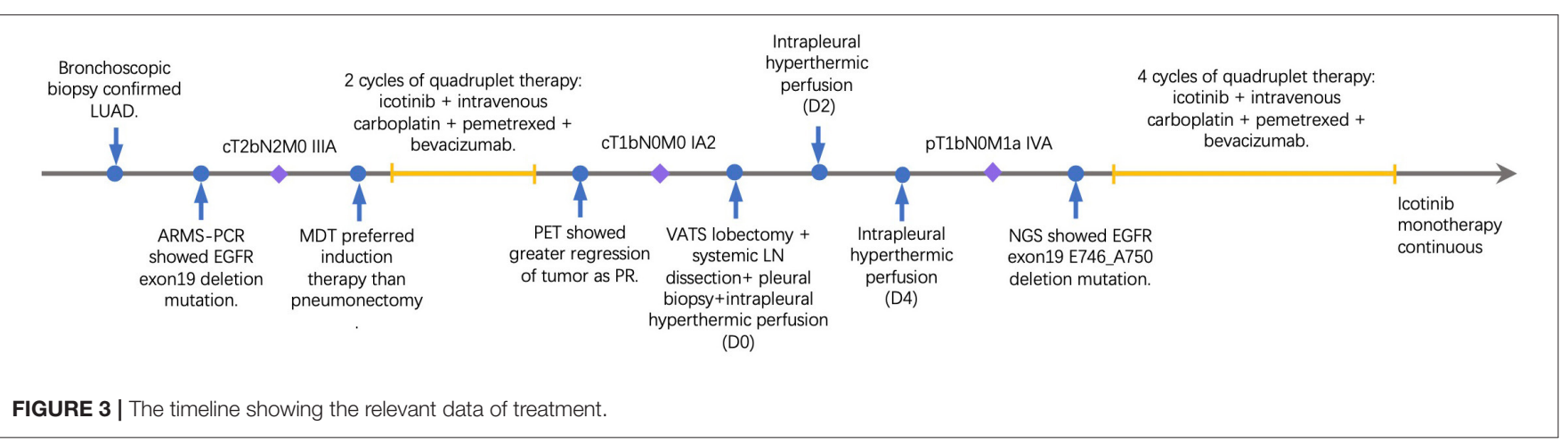

pleural metastasis was detected during surgery. In the operative fields, we observed tissue edema and hyperemia of the lung and moderate fibrosis of the external coat of vessels. The patient underwent a video-assisted thoracoscopic surgery (VATS) left lower lobectomy, systematic lymphadenectomy, pleural biopsy, and intrapleural hyperthermic perfusions, as well as two additional intrapleural hyperthermic perfusion performed on the next 2 days. The estimated intraoperative blood loss was nearly $200 \mathrm{ml}$ due to mild tissue hyperemia of the lung. The perioperative recovery was uneventful. The patient was discharged on the seventh postoperative day. Pathology confirmed the diagnosis of lung adenocarcinoma with pleural metastasis (ypT1bN0M1a, stage IVA). The histologic findings indicated significant tumor regression. It was characterized by an increase in the number of residue cancer cells, but fibrosis still predominated (Figures 2A,B). No lymph nodes metastasis was found at the dissected nodes station $5(0 / 1), 7(0 / 2)$, and $10(0 / 1)$; no vascular cancer thrombus and no residual tumor in the bronchial stumps were observed. The next-generation sequencing (NGS) of pathological specimens also confirmed EGFR 19 exon E746_A750 deletion mutation. Thus, after 1 month for recovery postoperatively, an additional of four-drug combination therapy was successfully conducted every 3 weeks \pm 3 day for four cycles. Single-agent icotinib was continued, with a follow-up chest CT scan every 3 months and PET/CT every 6 months. The latest follow-up recorded no disease progression in a chest CT scan when it was 16.7 months after surgery and 19.4 months after initial treatment. The timeline of treatment with relevant data of care was shown in Figure 3.

\section{DISCUSSION}

The patient's course illustrates the successful use of four-drug combination therapy to treat a locally advanced non-squamous NSCLC harboring EGFR mutation. The patient experienced significant tumor downstaging and underwent lobectomy instead of total pneumonectomy.

Patients with locally advanced NSCLC, especially N2 disease, should be considered for neoadjuvant chemotherapy or chemoradiotherapy based on NCCN guidelines. Although EGFR-TKI monotherapy is considered the standard first-line treatment for patients with TKI-sensitive EGFR mutations, most patients develop recurrence within $\sim 1$ year. The rationale for concurrent use of cytotoxic agents and bevacizumab is to reduce or even avoid potential de novo resistance (12). Sugawara (13) obtained the median progression-free survival (PFS) 18.3 and 15.3 months for the concurrent and sequential alternating regimens with gefitinib and carboplatin/pemetrexed in patients with EGFR-mutated NSCLC in a randomized phase II study, which is more promising in comparison to the PFS in previous studies with first-line gefitinib monotherapy (14). Two randomized clinical trials demonstrated erlotinib combined with bevacizumab harvest a median PFS of 16.0-16.9 months, which was significantly better than erlotinib monotherapy (7). 
Under the expectation to provide high-level, customized treatment for patients with resectable NSCLC, several phase II clinical trials had revealed the efficacy and safety on respect of preoperative TKI therapy $(10,11)$. EMERGING-CTONG 1103 showed the improved ORR for neoadjuvant erlotinib with $54.1 \%$ compared to gemcitabine plus cisplatin chemotherapy with $34.3 \%$ and doubling PFS with erlotinib (21.5 months) vs. GC chemotherapy (11.4 months; $p<0.001)$. Zhang's study showed similar ORR to the previous study, and patients with MPR were associated with improved survival while no patients reported grade 3 or 4 AEs. These results suggest that biomarkerguided neoadjuvant EGFR-TKI treatment in resectable NSCLC is promising. Hence, we combined these four drugs to optimize the treatment effect by avoiding potential de novo resistance. This case did reflect a promising effect. It is possible that TKI played a key role in the significant response of preoperative therapy. However, the first generation of EGFR-TKI is reversible TKI, which leaves it a tumor inhibitor. Chemotherapy provides a curative potential, which is critical for significant response of preoperative therapy and long-term tumor control. Since no neoadjuvant therapy can guarantee a full transfer to complete resection. In the long-term, however, bevacizumab may reduce potential de novo resistance if complete resection is unachievable. So it was important to include bevacizumab in the combination therapy.

Apart from a slight increase in intraoperative blood loss, there were no significant adverse events reported in this case, which was mainly the result of careful case selection and a fixed lower dosage of the cytotoxic agents and is far from a standard requirement. In our institute, we have attempt to apply the EGFR-TKI, EGFR-TKI/Chemotherapy, and Chemotherapy/Bevacizumab in several locally advanced NSCLC patients. The regimens of Chemotherapy were all adjusted to this fixed lower dosage. No significant adverse event was observed in the treatment period. All patients underwent surgery successfully. Yet, during the surgery, different degrees of tissue edema and hyperemia of the lung and fibrosis of the external coat of vessels in the operative fields. In general, any therapy involving EGFRTKI was expecting moderate to severe fibrosis of the external coat of vessels. There are a few reasons that accounted for the choice of icotinib in this patient. First, we did not choose erlotinib or gefitinib because there was a beneficence project in China so

\section{REFERENCES}

1. Bradley JD, Paulus R, Komaki R, Masters G, Blumenschein G, Schild $\mathrm{S}$, et al. Standard-dose versus high-dose conformal radiotherapy with concurrent and consolidation carboplatin plus paclitaxel with or without cetuximab for patients with stage IIIA or IIIB non-small-cell lung cancer (RTOG 0617): a randomised, two-by-two factorial phase 3 study. Lancet Oncol. (2015) 16:187-99. doi: 10.1016/S1470-2045(14)7 1207-0

2. Mazzoni F, Petreni P, Perna M, Scotti V, Bongiolatti S, Livi L, et al. Afatinib with subsequent surgery in stage III NSCLC with EGFR mutation: lessons learned from two clinical experiences. Tumor J. (2018) 104:P59. doi: $10.1177 / 0300891618775204$ that the patient can acquire a free prescription after 10 months of using icotinib. So it was more economical to use this drug, given the non-inferior to gefitinib in efficacy with favorable safety in non-selected or EGFR-mutant NSCLC patients (15). Second, we did not choose osimertinib because it would be 10 -fold higher than icotinib and nearly 20 times of cost if insurance was taken into consideration. Though median progression-free survival was significantly longer with osimertinib than SoC EGFR-TKI (16), the overall survival of the FLAURA study of osimertinib was not reported until recently (17).

In conclusion, treatment with this neoadjuvant combination therapy provided a novel therapeutic option for patients with locally advanced EGFR-mutated NSCLC facing total pneumonectomy.

\section{ETHICS STATEMENT}

The studies involving human participants were reviewed and approved by Ethics Committee of the First Affiliated Hospital of Guangzhou Medical University. Written informed consent was obtained from the individual(s) for the publication of any potentially identifiable images or data included in this article.

\section{AUTHOR CONTRIBUTIONS}

$\mathrm{JL}$ and $\mathrm{JH}$ was involved in planning and supervised the study. All authors wrote the manuscript and designed the figures. All authors contributed to the article and approved the submitted version.

\section{FUNDING}

This research was supported by the National Natural Science Foundation of China (grant 81703792), Guangzhou Science and Technology Programs for Science Study (grant 201804010052), and Project of State Key Laboratory of Respiratory Disease (grant SKLRD-QN-201706).

\section{ACKNOWLEDGMENTS}

We would like to express our heartfelt gratitude to Lindsey Hamblin for her English proof reading.

3. Yang JC, Srimuninnimit V, Ahn MJ, Lin CC, Kim SW, Tsai CM, et al. First-line pemetrexed plus cisplatin followed by Gefitinib maintenance therapy versus Gefitinib monotherapy in East Asian never-smoker patients with locally advanced or metastatic nonsquamous non-small cell lung cancer: final overall survival results from a randomized phase 3 study. J Thorac Oncol. (2016) 11:370-9. doi: 10.1016/j.jtho.2015.11.008

4. Noronha V, Patil VM, Joshi A, Menon N, Chougule A, Mahajan A, et al. Gefitinib versus gefitinib plus pemetrexed and carboplatin chemotherapy in EGFR-mutated lung cancer. J Clin Oncol. (2020) 38:124-36. doi: 10.1200/JCO.19.01154

5. Yokoi T, Torii Y, Katashiba Y, Sugimoto H, Tanijiri T, Ogata M, et al. Phase II study of pemetrexed and carboplatin plus bevacizumab, followed by maintenance pemetrexed and bevacizumab in Japanese patients with 
non-squamous non-small cell lung cancer. Oncol Lett. (2014) 8:2453-7. doi: 10.3892/ol.2014.2552

6. Nakagawa K, Garon EB, SetoT, Nishio M, Aix SP, Paz-Ares L, et al., Ramucirumab plus erlotinib in patients with untreated, EGFR-mutated, advanced non-small-cell lung cancer (RELAY): a randomised, doubleblind, placebo-controlled, phase 3 trial. Lancet Oncol. (2019) 20:1655-69. doi: 10.1016/S1470-2045(19)30634-5

7. Saito H, Fukuhara T, Furuya N, Watanabe K, Sugawara S, Iwasawa S, et al. Erlotinib plus bevacizumab versus erlotinib alone in patients with EGFR-positive advanced non-squamous non-small-cell lung cancer (NEJ026): interim analysis of an open-label, randomised, multicentre, phase 3 trial. Lancet Oncol. (2019) 20:625-35. doi: 10.1016/S1470-2045(19)30035-X

8. Kurata T, Nakaya A, Yokoi T, Niki M, Kibata K, Takeyasu Y, et al. Phase I/II study of erlotinib, carboplatin, pemetrexed, and bevacizumab in chemotherapy-naïve patients with advanced non-squamous non-small cell lung cancer harboring epidermal growth factor receptor mutation. Genes Cancer. (2017) 8:559-65. doi: 10.18632/genesandcancer.145

9. Xiong L, Li R, Sun J, Lou Y, Zhang W, Bai H, et al. Erlotinib as neoadjuvant therapy in stage IIIA (N2) mutation-positive non-small cell lung cancer: a prospective, single-arm, phase II study. Oncologist. (2019) 24:157-64. doi: 10.1634/theoncologist.2018-0120

10. Zhong WZ, Chen KN, Chen C, Gu CD, Wang J, Yang XN, et al. Erlotinib versus gemcitabine plus cisplatin as neoadjuvant treatment of stage IIIA-N2 -mutant non-small-cell lung cancer (EMERGING-CTONG 1103): a randomized phase II study. J Clin Oncol. (2019) 37:2235-45. doi: 10.1200/JCO.19.00075

11. Zhang Y, Fu F, Hu H, Wang S, Li Y, Hu H, et al. Gefitinib as neoadjuvant therapy for resectable stage II-IIIA non-small cell lung cancer: a phase II study. J Thorac Cardiovasc Surg. (2020). doi: 10.1016/j.jtcvs.2020.02.131. [Epub ahead of print].

12. Takahashi K, Saito H. Is epidermal growth factor receptor tyrosine kinase inhibitor in combination with cytotoxic chemotherapy a better treatment option for patients with EGFR-mutated non-small-cell lung cancer? Transl Lung Cancer Res. (2016) 5:98-101. doi: 10.3978/j.issn.2218-6751.2015.08.14

13. Sugawara S, Oizumi S, Minato K, Harada T, Inoue A, Fujita $Y$, et al. Randomized phase II study of concurrent versus sequential alternating gefitinib and chemotherapy in previously untreated non-small cell lung cancer with sensitive EGFR mutations: NEJ005/TCOG0902. Ann Oncol. (2015) 26:888-94. doi: 10.1093/annonc/mdv063

14. Maemondo M, Inoue A, Kobayashi K, Sugawara S, Oizumi S, Isobe H, et al. Gefitinib or chemotherapy for non-small-cell lung cancer with mutated EGFR. N Engl J Med. (2010) 362:2380-8. doi: 10.1056/NEJMoa0909530

15. Shi Y, Zhang L, Liu X, Zhou C, Zhang L, Zhang S, et al. Icotinib versus gefitinib in previously treated advanced non-small-cell lung cancer (ICOGEN): a randomised, double-blind phase 3 non-inferiority trial. Lancet Oncol. (2013) 14:953-61. doi: 10.1016/S1470-2045(13)70355-3

16. Soria JC, Ohe Y, Vansteenkiste J, Reungwetwattana T, Chewaskulyong B, Lee $\mathrm{KH}$, et al. Osimertinib in untreated EGFR-mutated advanced non-small-cell lung cancer. N Engl J Med. (2018) 378:113-25. doi: 10.1056/NEJMoa1713137

17. Ramalingam SS, Vansteenkiste J, Planchard D, Cho BC, Gray JE, Ohe Y, et al. Overall survival with osimertinib in untreated, EGFR-mutated advanced NSCLC. N Engl J Med. (2020) 382:41-50. doi: 10.1056/NEJMoa1913662

Conflict of Interest: The authors declare that the research was conducted in the absence of any commercial or financial relationships that could be construed as a potential conflict of interest.

Copyright (c) $2020 \mathrm{Li}, \mathrm{Xu}$, Cai, Zhang, Zeng, Cui, Hao, Liu and He. This is an open-access article distributed under the terms of the Creative Commons Attribution License (CC BY). The use, distribution or reproduction in other forums is permitted, provided the original author(s) and the copyright owner(s) are credited and that the original publication in this journal is cited, in accordance with accepted academic practice. No use, distribution or reproduction is permitted which does not comply with these terms. 